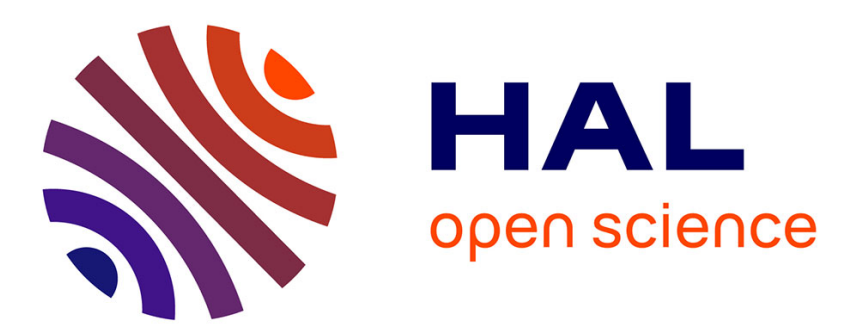

\title{
Theoretical Demonstration of the Potentiality of Boron Nitride Nanotubes to Encapsulate Anticancer Molecule
}

Mohamed El Khalifi, Eric Duverger, Hatem Boulahdour, Fabien Picaud

\section{To cite this version:}

Mohamed El Khalifi, Eric Duverger, Hatem Boulahdour, Fabien Picaud. Theoretical Demonstration of the Potentiality of Boron Nitride Nanotubes to Encapsulate Anticancer Molecule. Physical Chemistry Chemical Physics, 2015, 17 (44), pp.30057 - 30064. hal-02868340

\author{
HAL Id: hal-02868340 \\ https://hal.science/hal-02868340
}

Submitted on 15 Jun 2020

HAL is a multi-disciplinary open access archive for the deposit and dissemination of scientific research documents, whether they are published or not. The documents may come from teaching and research institutions in France or abroad, or from public or private research centers.
L'archive ouverte pluridisciplinaire HAL, est destinée au dépôt et à la diffusion de documents scientifiques de niveau recherche, publiés ou non, émanant des établissements d'enseignement et de recherche français ou étrangers, des laboratoires publics ou privés. 


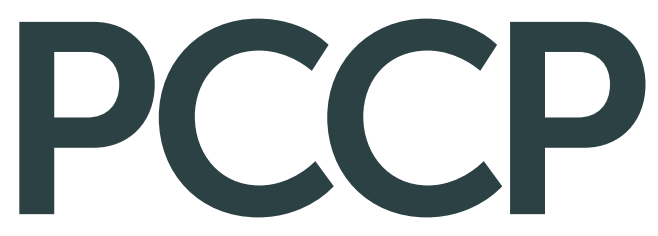

Accepted Manuscript

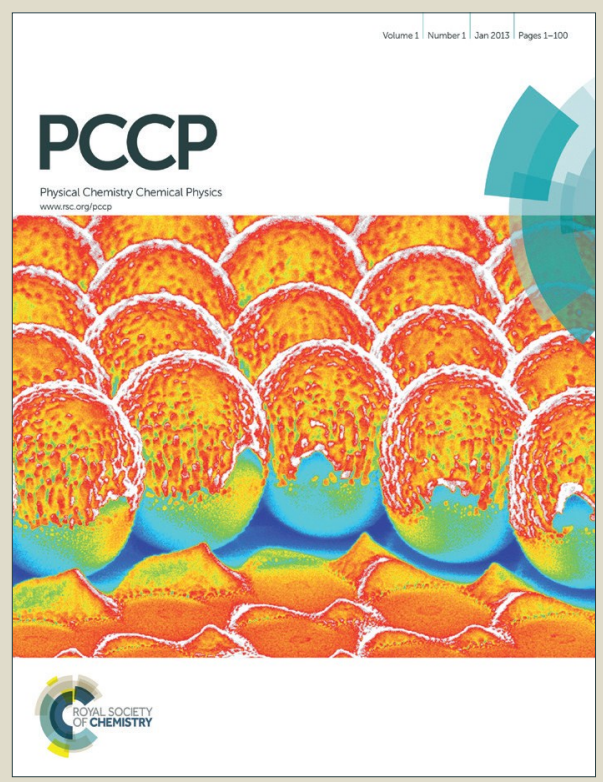

This is an Accepted Manuscript, which has been through the Royal Society of Chemistry peer review process and has been accepted for publication.

Accepted Manuscripts are published online shortly after acceptance, before technical editing, formatting and proof reading. Using this free service, authors can make their results available to the community, in citable form, before we publish the edited article. We will replace this Accepted Manuscript with the edited and formatted Advance Article as soon as it is available.

You can find more information about Accepted Manuscripts in the Information for Authors.

Please note that technical editing may introduce minor changes to the text and/or graphics, which may alter content. The journal's standard Terms \& Conditions and the Ethical guidelines still apply. In no event shall the Royal Society of Chemistry be held responsible for any errors or omissions in this Accepted Manuscript or any consequences arising from the use of any information it contains. 


\title{
Theoretical Demonstration of the Potentiality of Boron Nitride Nanotubes to Encapsulate Anticancer Molecule.
} M. El Khalifi ${ }^{a}$, E. Duverger ${ }^{b}$, T. Gharbi ${ }^{a}$, H. Boulahdour ${ }^{a}$ and F. Picaud ${ }^{a, *}$.

${ }^{a}$ Laboratoire de Nanomédecine, Imagerie et Thérapeutique, Université Franche-Comté (UFR Sciences et Techniques), Centre Hospitalier Universitaire de Besançon, 16 route de Gray, 25030 Besançon, France

${ }^{\mathrm{b}}$ Institut FEMTO-ST, 32 Avenue de l'Observatoire, 25044 Besançon, France *corresponding author : email: fabien.picaud@univ-fcomte.fr. Phone number: +33381666284

\begin{abstract}
Anticancer drug transport is now becoming an important scientific challenge since it would allow localizing the drug release near the tumor cell, avoiding secondary medical effects. We present theoretical results, based on density functional theory and molecular dynamics simulations, which demonstrate the stability of functionalized single $(10,10)$ boron nitride nanotubes (BNNTs) filled with anticancer molecule such as carboplatin (CPT). For this functionalized system we determine the dependence of the adsorption energy on the molecule displacement near the inner BNNTs surface, together with their local morphological and electrical changes and compare the
\end{abstract}


values to the adsorption energy obtained on the outer surface. Quantum simulations show that the most stable physisorption state is located inside the nanotube, with no net charge transfer. This demonstrates that chemotherapeutic encapsulation is the most favorable way to transport drug molecules. The solvent effect and dispersion repulsion contributions are then taken into account using molecular dynamics simulations. Our results confirm that carboplatin therapeutic agents are not affected when they were adsorbed inside BNNTs by the surrounding water molecules.

Keywords: boron nanotubes; therapeutic agents; DFT calculations; Molecular Dynamic

\section{Introduction}

Chemotherapy is frequently used to treat cancer patients. However, serious adverse effects are observed when chemotherapeutic agents are systemically administered since they exhibit poor specificity in reaching tumor tissues. ${ }^{1}$ More, the efficacy of many potent and promising drug molecules is limited by their low water solubility, by the increased drug resistance and highly cytotoxic side effects. ${ }^{2}$ To circumvent such important drawbacks, an efficient way of systemic transportation needs to be developed in order to improve the pharmacological properties of conventional drugs. ${ }^{3}$ Incorporating chemotherapeutics drug and receptors into a nanocarrier is a promising way to develop since it could protect the active molecule from solvent despite some drawbacks. ${ }^{4-7}$ Indeed, the random nature of the targeting makes difficult to control the transport process and the appropriate drug release. This lack of control could be at the origin of the multiple drug resistance that could be overcome using programmed nanocarriers to actively bind specific cells. This binding may be achieved using a targeting agent that interacts specifically with a receptor overexpressed on the cancer cells. ${ }^{8}$ 
In recent years, various types of drug/agent nanocarriers have been investigated, among which a small number of such systems are already commercialized or are in clinical studies. ${ }^{9}$ In particular, carbon nanotubes CNTs have attracted a wide attention as carriers for biologically relevant molecules, because of their unique physicochemical and biological properties. ${ }^{7,}$ 10, 11 Among all the nanocarriers studied, BNNTs are structural analogues of CNTs nanomaterials having a band gap equal to $5.5 \mathrm{eV}^{12}$ (independent of nanotube diameter and chirality). Moreover they exhibit significant interesting properties compared to CNTs with regard to superior chemical and thermal stability and excellent piezoelectric properties. Using noncovalent polymer wrapping, the biocompatibility and solubility issues of BNNTs can be resolved, enabling studies on their interactions with the human cells. ${ }^{13}$ Xie et al. first reported the development of a soluble BNNT via functionalization with amine-terminated oligomeric polyethylene glycol. ${ }^{14}$ DFT studies on the noncovalent functionalization of BNNTs with benzaldehyde and seven different heterocyclic aromatic molecules, showed that weak interactions give rise to new impurity states within the band gap of pristine BNNTs suggesting the possibility of carrier doping through the selective adsorption of aromatic rings. ${ }^{15}$ Recently we studied the interactions between BNNTs and different molecules, such as azomethine $\left(\mathrm{C}_{2} \mathrm{H}_{5} \mathrm{~N}\right)$ and an anticancer agent (Pt(IV) complex) linked to an amino-derivative chain. Our results showed that the most stable physisorption state for both molecules was located inside the nanotube and that the molecular chemisorption was possible only when the azomethine was present above two adjacent $\mathrm{B}$ and $\mathrm{N}$ atoms of a hexagon. The attachment of an azomethine plus a subsequent drug did not perturb the cycloaddition process. ${ }^{16}$ Moreover, some preliminary applications in biomedicine have emerged in the latest years showing no significant adverse effects for in vivo study when BNNTs were injected in 
rabbits at a dose up to $10 \mathrm{mg} / \mathrm{kg}$. All these data suggested the optimal biocompatibility of BNNTs, and thus enabling their exploitation in nanomedecine. ${ }^{17,18}$

In this paper we report some new theoretical results concerning the adsorption of Carboplatin (CPT) molecule (a chemotherapeutic agent) outside and inside BNNTs nanocarriers. Thanks to full DFT calculations we determine the route to stabilize this anticancer molecule inside BNNTs, and we demonstrate that carboplatin can find stable geometry leading to strong adsorption onto $(10,10)$ BNNT sidewalls. To demonstrate this, we investigated the stability of the functionalized systems through the changes of its morphology and its electronic structure. To take into account van der Waals interactions and solvent effects in such biologic systems, we have conducted allatom molecular dynamics simulations on the carboplatin encapsulation phenomena. They supported the DFT results for the anticancer molecules confinement, even in the presence of water solvent.

\section{Materials and Methods}

\section{a) Quantum simulations}

While carbon nanotubes are ended in equal proportion by zigzag, armchair or chiral structures, the BNNT ended structures are fully hooked on the synthesis and development methods. As pointed in several works, while dominant BNNT ended are zigzag, BNNTs grown directly from the vapor phase adopted preferentially an armchair configuration while those grown from laserheating and carbon substituted methods are zigzag type. ${ }^{19,}{ }^{20}$ We will study here system consisting of an armchair single-walled $(10,10)$ BNNT with finite-length cylindrical BN cage (i.e. diameter radius $13.94 \AA$, length $21.32 \AA$ ), composed of $180 \mathrm{~B}$ and $180 \mathrm{~N}$ atoms. In order to avoid boundary effects, hydroxyl groups $(-\mathrm{OH})$, consisting of $40 \mathrm{O}$ and $40 \mathrm{H}$ atoms, were 
introduced onto the BNNT extremities, as depicted in Figure 1. These functions were usually applied to increase the BNNT dispersion in solvent and hold out their field of research in medicine and biomedical applications via covalent attachment. ${ }^{21} \mathrm{We}$ can also notice that $-\mathrm{COOH}$ groups can be equally held on the dangling bonds of nanotubes. ${ }^{22}$ Carboplatin $\left(\mathrm{C}_{6} \mathrm{H}_{12} \mathrm{~N}_{2} \mathrm{O}_{4} \mathrm{Pt}\right)$ drug molecule was studied in the gas phase with the same unit cell of the BNNT tube (i.e. 50 x 50 x 60) $\AA^{3}$, where the last number represented the length of the unit cell along the tube axis. Given the large unit cell, the Brillouin zone was sampled using a single k-point at the center $\Gamma$. A basis set of localized atomic orbitals (double- $\zeta$ plus polarization functions), and norm-conserving pseudopotentials were employed.

The adsorption energy $\left(E_{a d s}\right)$ of adsorbed molecule (Mol) on the inner (or outer) surface of the BNNT (Template) was derived from the energy difference between the different states of the system, namely, $\mathrm{E}_{\mathrm{ads}}=\mathrm{E}(\mathrm{Mol}+$ Template $)-\mathrm{E}($ Template $)-\mathrm{E}(\mathrm{Mol})$. A negative $\mathrm{E}_{\mathrm{ads}}$ value denoted a more favorable interaction between the drug and the BNNT.

The total energies were obtained from ab initio calculations in the framework of Kohn-Sham realization of the density functional theory DFT. $^{23,24}$ We used the generalized gradient approximation (GGA) in the form proposed by Perdew et al. ${ }^{25}$ for the exchange correlation density functional as implemented in the SIESTA package. ${ }^{26}$ All calculations were performed without spin polarization. We use the self-consistency mixing rate of 0.1 , a maximum force tolerance of $0.01 \mathrm{eV} / \AA$ and a mesh cutoff of $100 \mathrm{Ry}$ (the variations of these parameters showed a very low perturbation of the total energies by less than $0.1 \%$ ). The self-consistent cycles were stopped when variations of the total energy per unit cell and band structure energy were both less than $10^{-4} \mathrm{eV}$.

\section{b) Molecular dynamics simulations}


Dynamic molecular simulations and minimization was performed using NAMD 2.9b2 package. The CHARMM36 force field parameters and TIP3P water model are used in all simulations. ${ }^{27} \mathrm{~A}$ temperature of $300 \mathrm{~K}$ (Langevin Dynamics) and a pressure of $1 \mathrm{~atm}$ (Langevin piston) of the system were kept constant during simulation. The long range electrostatic forces were measured by applying the classical particle mesh Ewald (PME) method with a grid spacing of $1.2 \AA$ and a fourth-order spline interpolation. The integration time step was equal to $1 \mathrm{fs}$.

Each simulation employs periodic boundary conditions in the three directions of space and Particle Mesh Ewald is used to calculate electrostatic interactions. The BNNTs are built in the same way than carbon nanotube using a boron-nitrogen bond distance of $1.446 \AA$. The partial charges for the boron and the nitrogen atoms of the BNNTs were taken from the Bader analysis of our theoretical calculations. The CPT molecule force field was established according to the force field toolkit package of visual molecular dynamic program. ${ }^{28}$

\section{Results and discussion}

a)

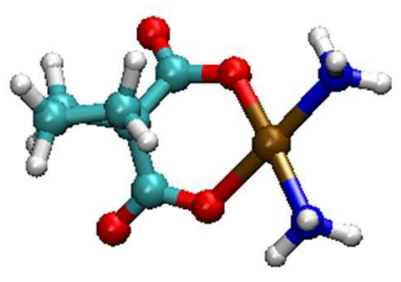

b)

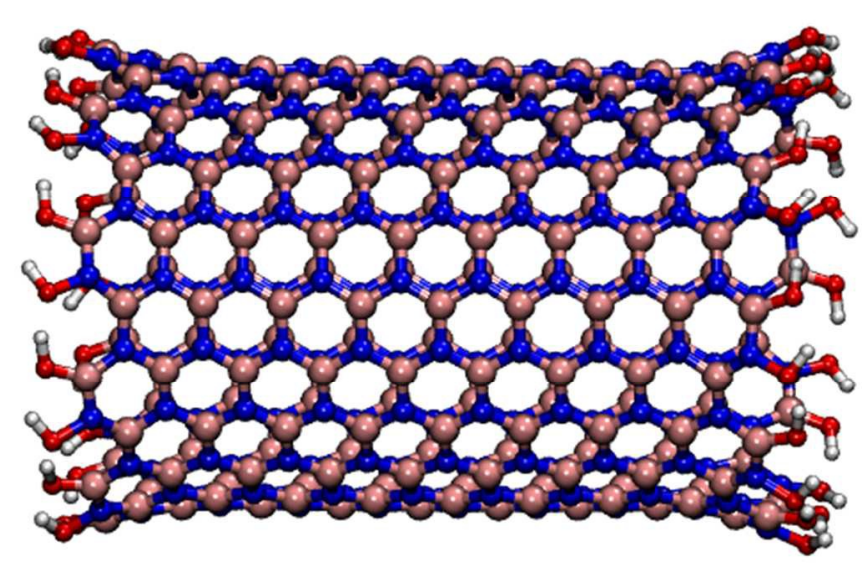


Figure 1: Geometry of (a) Carboplatin $\left(\mathrm{C}_{6} \mathrm{H}_{12} \mathrm{~N}_{2} \mathrm{O}_{4} \mathrm{Pt}\right)$, (b) single-walled $(10,10)$ BNNTs with hydroxyl groups $(-\mathrm{OH})(\mathrm{B}, \mathrm{N}, \mathrm{C}, \mathrm{O}, \mathrm{H}, \mathrm{Pt}$ and $\mathrm{Cl}$ atoms are represented as pink, blue, small cyan, red, white, tan, large cyan spheres, respectively).

\subsection{Carboplatin}

Carboplatin is a current chemotherapy drug used against several types of cancer. Since its introduction in the late 1980s, it became popular in clinical treatment due to its vastly reduced side-effects. $^{29}$ It corresponds to the second generation of platinum agent showing higher solubility in water and fewer side-effects than its parent agent (cisplatin). ${ }^{30}$

a) Adsorption on outer surface

To examine the interaction between the drug molecule and its carrier, we first placed it on the outer surface of the BNNT. Indeed, this adsorption site will be always accessible for the molecule while the inner surface is related to the aperture of the pore. The optimization of the molecule geometry shows that the molecule tends to lie flat on the surface with an energy minimum equal to $-0.29 \mathrm{eV}$. This is sufficiently low to stabilize the drug on the surface, but maybe, not enough to hold the molecule on the BNNT surface as it will be transferred close to the cancer cell.

b) Drug encapsulation

The investigation of CPT interaction with the inner surface of BNNTs is realized in considering two different models. In the first one, we study the molecule centered along the main axis of the BNNT (symmetric position). In the second one, the molecule is put near the inner surface of the BNNT. In all simulations, the molecule positions were optimized. The mean distance between the Pt atom of the molecule and the BNNT center of mass, denoted as d, was calculated. Then, the position of the molecule was progressively changed along the tube axis in order to determine the interaction molecule-BNNT with d varying from $-21 \AA$ to $21 \AA$. For each distance, the whole 
structure was relaxed by allowing atom displacement to minimize the total energy. The adsorption energy of the interacting system is obtained by subtracting both the total energy of the isolated tube and the energy of the molecule in the gas phase to the total energy of the combined structure after optimization. Figure 2 shows the adsorption energy of the CPT molecule as a function of the distance $d$ for the two models envisioned.

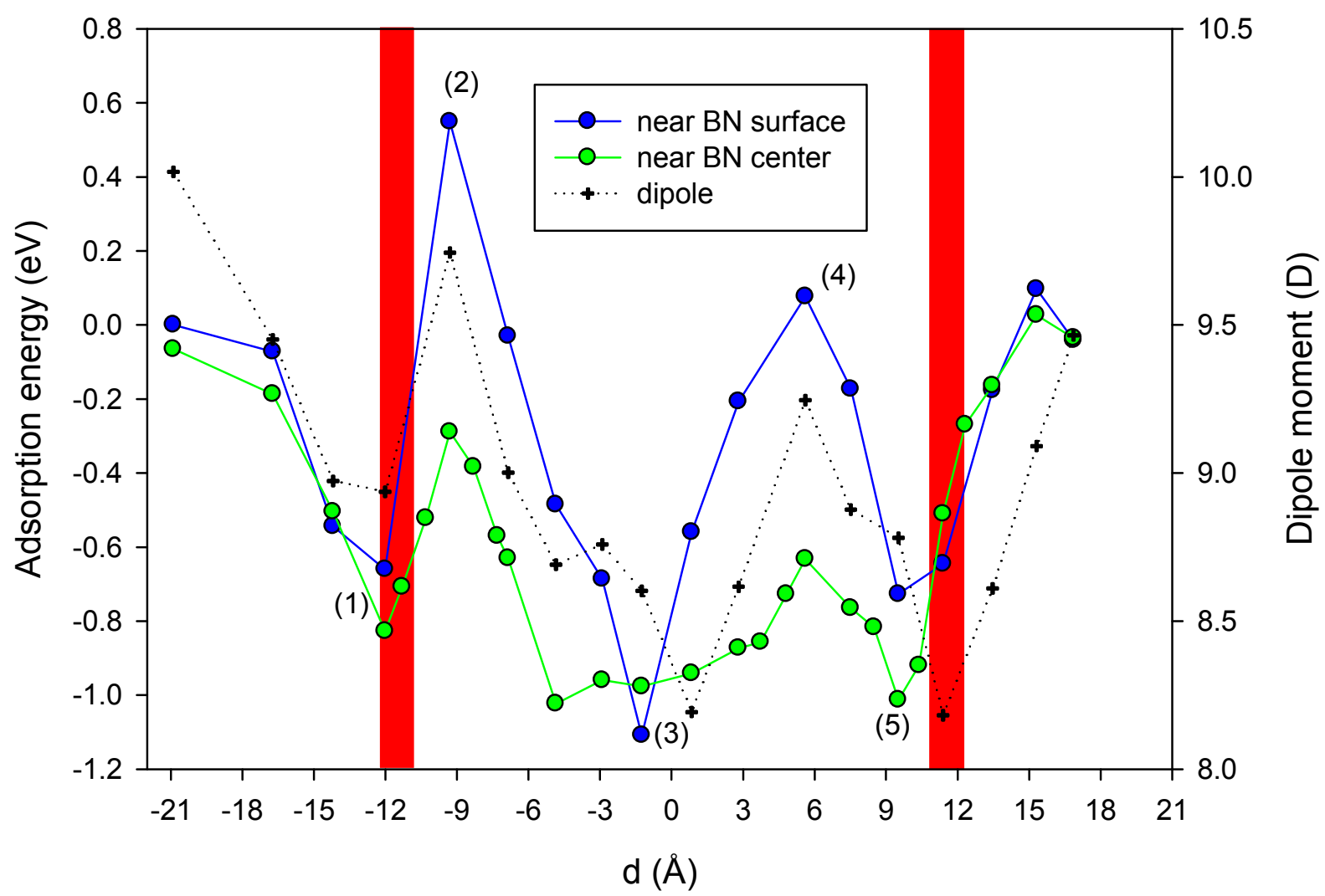

Figure 2: interaction energy of CPT as a function of d, defined as the distance between the absolute position of Pt atom in the CPT molecule and the center of mass of BNNT. (Green curve: symmetric position of CPT with respect to the nanocarrier wall; blue curve: CPT close to the BNNT wall). Black dotted curve represents the evolution of the total dipole moment during the encapsulation of the molecule. Red bars highlight the BNNT edges. 
For the first model considered, as shown in Figure 2, the interaction molecule-BNNT presents several characteristics. When the molecule is approaching the nanotube entrance (i.e. $d$ negative), the adsorption energy is decreasing from -0.01 to $-0.78 \mathrm{eV}$. This is followed by a high-energy barrier to enter the inner part of the BNNT (height $=0.78 \mathrm{eV} ; \mathrm{d}=-9 \AA$ ), centered on the hydroxyl groups, which are in interaction with the carbon and oxygen of the molecule. Then we can observe the formation of an important attractive and large valley (around $-1.0 \mathrm{eV}$ ) with one minimum at $-1.15 \mathrm{eV}$ near the BNNT center of mass $(\mathrm{d}=0)$, corresponding to the most stable adsorption site for this study. For larger d ( $\mathrm{d}$ ranges from 0 to $9 \AA$ ), another potential barrier with a height equal to $0.4 \mathrm{eV}$ appears. On the exterior of the nanocarrier, we see a third potential barrier largely outside the BNNT edge corresponding to the passage of the molecular carbon ring. We can note that the energy diffusion valley is dissymmetric due to the asymmetry of the CPT. All these results show that the molecule can be confined inside the BNNT. Indeed, in this geometry, it should raise an important energy barrier to exit from the nanocarrier.

For the second model, with the molecule near the BNNT inner surface, the interaction energy modifies strongly its shape. We can always observe adsorption energy well equal to $-1.15 \mathrm{eV}$ at the same $d$ distance that in the first model. We retrieve three potential barriers inside and outside the nanotube. The potential barrier height at the nanopore entrance is increased highlighting a strong repulsive interaction between the molecule and the inner surface. In the same way, the second potential barrier between $d=0 \AA$ and $d=9 \AA$ is strongly increased. On the contrary, the third potential barrier at the exit edge of the BNNT nanotube is not modified in shape and height. We have performed the same calculations using $-\mathrm{H}$ (or $-\mathrm{COOH}$, respectively) functional to saturate the dangling bonds. Our calculations demonstrate that no potential barrier $(0.11 \mathrm{eV}$ (or 
$0.04 \mathrm{eV}$ respectively)) appears using these functional while the exit barrier remains almost unchanged (0.94 eV (or $0.83 \mathrm{eV}$ respectively)).

These two energy adsorption paths permit to have a hint of the total diffusion path across the BNNT that is thus the combination of the two curves shown in Figure 2. We can assume that in general, the molecule tends to stay close to the center of the BNNT. Note that the same calculations were performed on zigzag BNNT of length $23.6 \AA$ and diameter $14.9 \AA$. The main difference observed during the CPT encapsulation was the disappearance of the energy barrier at the entrance of the tube which could favor the encapsulation velocity of the molecule in experiments. This comes from the number of $\mathrm{OH}$ functions (17 in the zigzag case compared to 20 in the armchair case) that improves the molecule confinement.

To quantify the modifications submitted to the molecule during its encapsulation, we analyze the projected electronic densities of states (PDOS) and the variation of Bader charges in three important positions. The first one is at the entrance of the BNNT, the second at the top of the potential barrier, the last one in the potential well. They are denoted as (1), (2) and (3), in Figure 2 respectively. The same characteristics were observed in the energy peak observed for $\mathrm{d}>0$. 
Bader Total Charge on isolated CPT: $80.00 \mathrm{e}$

Total Dipole on Isolated systems: 9.72D $\quad-0.91 \mathrm{D} \quad 2.11 \mathrm{D}$

(1)

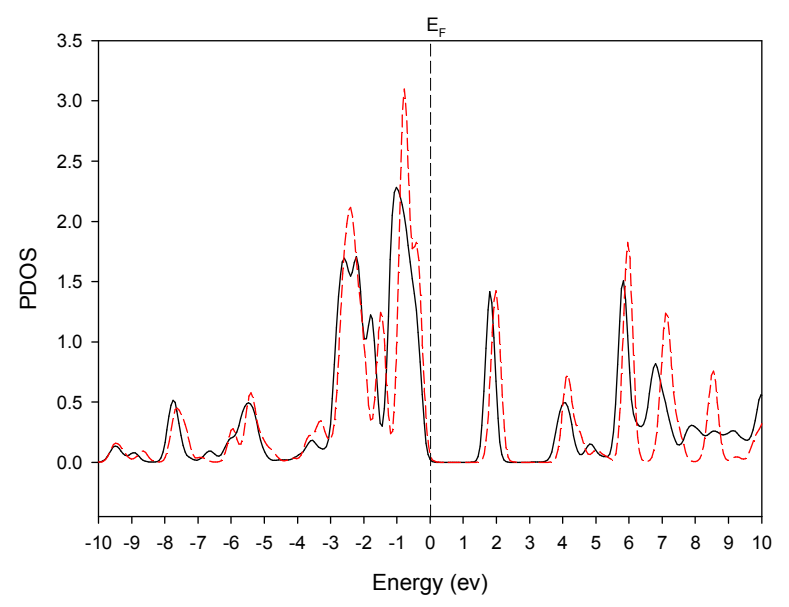

\begin{tabular}{|c|c|c|c|c|}
\hline \multicolumn{5}{|c|}{ Bader charge variation (e) of atom } \\
\hline $\mathrm{Pt}$ & $\mathrm{N}$ & $\mathrm{C}$ & $\mathrm{O}$ & $\mathrm{H}$ \\
\hline-0.03 & 0.32 & -0.16 & $\mathbf{0 . 0 7}$ & -0.01 \\
\hline \multicolumn{5}{|c|}{ Dipole (D) } \\
\hline \multicolumn{2}{|c|}{$\mathrm{X}$} & \multicolumn{2}{|c|}{ Y } & $\mathrm{Z}$ \\
\hline \multicolumn{2}{|c|}{9.00} & \multicolumn{2}{|c|}{-0.16} & 1.43 \\
\hline \multicolumn{5}{|c|}{ Bader Total Charge on CPT (e): $\mathbf{8 0 . 0 4}$} \\
\hline
\end{tabular}

(2)

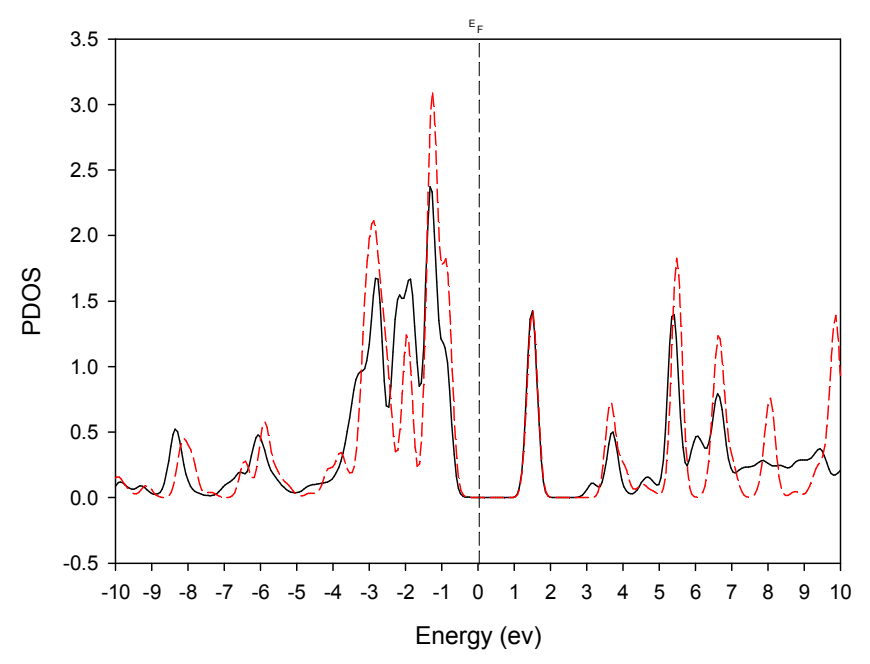

Bader charge variation (e) of atom

\begin{tabular}{|l|l|l|l|l}
\hline $\mathrm{Pt}$ & $\mathrm{N}$ & $\mathrm{C}$ & $\mathrm{O}$ & $\mathrm{H}$ \\
\hline $\mathbf{0 . 0 6}$ & $\mathbf{- 0 . 5 3}$ & $\mathbf{- 0 . 1 1}$ & $\mathbf{- 0 . 0 9}$ & $\mathbf{0 . 1 5}$ \\
\hline
\end{tabular}

Dipole (D)

\begin{tabular}{|c|c|c|}
\hline $\mathrm{X}$ & $\mathrm{Y}$ & $\mathrm{Z}$ \\
\hline $\mathbf{9 . 4 0}$ & $\mathbf{0 . 1 5}$ & $\mathbf{1 . 0 3}$ \\
\hline
\end{tabular}

Bader Total Charge on CPT (e): 79.96 
(3)

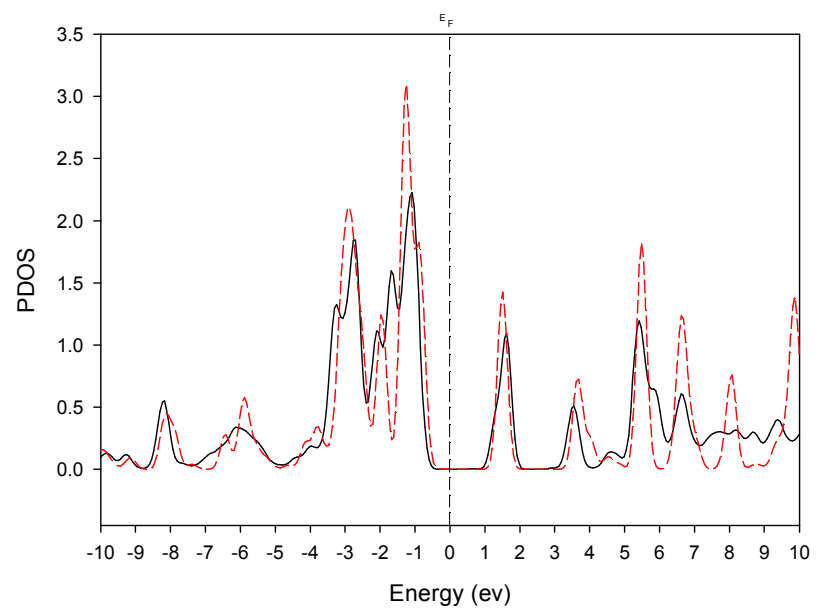

\begin{tabular}{|l|l|l|l|l|}
\hline \multicolumn{3}{|c|}{ Bader charge variation (e) of atom } \\
\hline Pt & N & C & O & H \\
\hline $\mathbf{0 . 0 2}$ & $\mathbf{- 0 . 5 8}$ & $\mathbf{0 . 0 8}$ & $\mathbf{- 0 . 0 4}$ & $\mathbf{0 . 0 2}$ \\
\hline \multicolumn{5}{|c|}{ Dipole (D) } \\
\hline X & Y & Z \\
\hline $\mathbf{8 . 7 8}$ & $\mathbf{0 . 1 7}$ & $\mathbf{1 . 2 7}$ \\
\hline Bader Total Charge on CPT (e): 79.57 \\
\hline
\end{tabular}

Figure 3: Projected electronic density of states of the CPT in the three positions depicted in Figure 2: (1) well at the entrance of BNNT; (2) barrier and (3) most stable site in the BNNT together with those of the molecule alone (red curves). Fermi level was placed at $0 \mathrm{eV}$. The tables show the mean variations of the Bader charges of the CPT atoms and the total electric dipole of the system, depending on the positions of the molecules. Bader Total charge summation on the molecules is also indicated in the table, as well as the total dipole when the molecule and BNNT are far away.

As sketched in Figure 3, the projected density of states (PDOS) for the three positions was slightly changing but was showing the same features. We retrieve an energy gap equal to $1.5 \mathrm{eV}$ whatever the positions of the drug inside the BNNT (1.4 eV in position 1 and $1.5 \mathrm{eV}$ for positions 
2 and 3). The distribution of the Bader charges presents some local changes on the atoms. However we did not observe any significant changes of the total molecular charge during the encapsulation of the molecule compared to the gas phase. We assume that these small charge variations are due to electrostatic interactions between the molecule and the BNNT inner surface without any chemical functionalization. If we are interested now in the amount of the charges loaded on the CPT when encapsulated compared to it alone, we observe a slight charge transfer from the molecule to the BNNT during its energy path. The most important charge transfer was held when the molecule is close to the position (3) mainly due to charge modification on $\mathrm{O}, \mathrm{N}$ and Pt atoms of the molecule. This could be understandable since the position (3) corresponds to the passage of the last $-\mathrm{CO}$ and $-\mathrm{NH}_{2}$ groups through the $\mathrm{BNNT}$, while the $\mathrm{Pt}$ atom is still inside the nanotube.

To interpret the apparition of the different zones in the energy path, we investigate the total dipole moment value. It is mainly due to the dipole of the drug molecule since the BNNT alone presents a dipole moment close to $1 \mathrm{D}$ while the CPT presents a dipole of 13D, whose main component is well oriented along the $\mathrm{Pt}-\mathrm{C}$ bond. From a general point of view the total dipole moment was rather stable during the molecule encapsulation. However, due to the molecule asymmetry, the dipole component along the BNNT axis (here, the $\mathrm{x}$ axis) shows the same behavior as the adsorption energy and varies according to the molecular position. In position (1), the main dipole component is equal to $9 \mathrm{D}$. Then it increases until 9.4D in position (2), to decrease at $8.78 \mathrm{D}$ in position (3) as the energy path. The following diffusion sites are likewise characteristic of the dipole moment since the second barrier (position (4)) leads to a dipole moment of $9.25 \mathrm{D}$ to finally decrease until $8.28 \mathrm{D}$ in the last energy well (position (5)), before the release of the drug molecule. The diffusion path of the encapsulated molecule will be thus 
fundamentally governed by the total dipole moment along the tube as sketched by the dotted line in Figure 2. To visualize the physical interactions, we show in Figure 4 the electron localization function (ELF) representation of the system at positions (1) to (3) of Figure 2. This function produces informative patterns and describes chemical bonding in molecules and solids. It measures the probability of finding an electron near another electron with the same spin related to the Pauli Exclusion Principle. ${ }^{31}$ The upper limit of the ELF representation corresponds to chemical bonding while the values lower than 0.5 correspond to electron-gas-like pair probability (i.e. no chemical bonding). As demonstrated in Figure 4, no chemical interaction was involved in the strong confinement of the CPT inside the BNNT. Note that the same calculations were performed on carbon nanotube with corresponding geometric parameters. ${ }^{32}$ The energy barrier to enter the nanotube was very small $(0.07 \mathrm{eV})$ while the inner site was characterized by an energy well close to $-0.55 \mathrm{eV}$. The release of the CPT in such well known nanostructure is thus easier and could be too fast in contrary to BNNT nanotube.

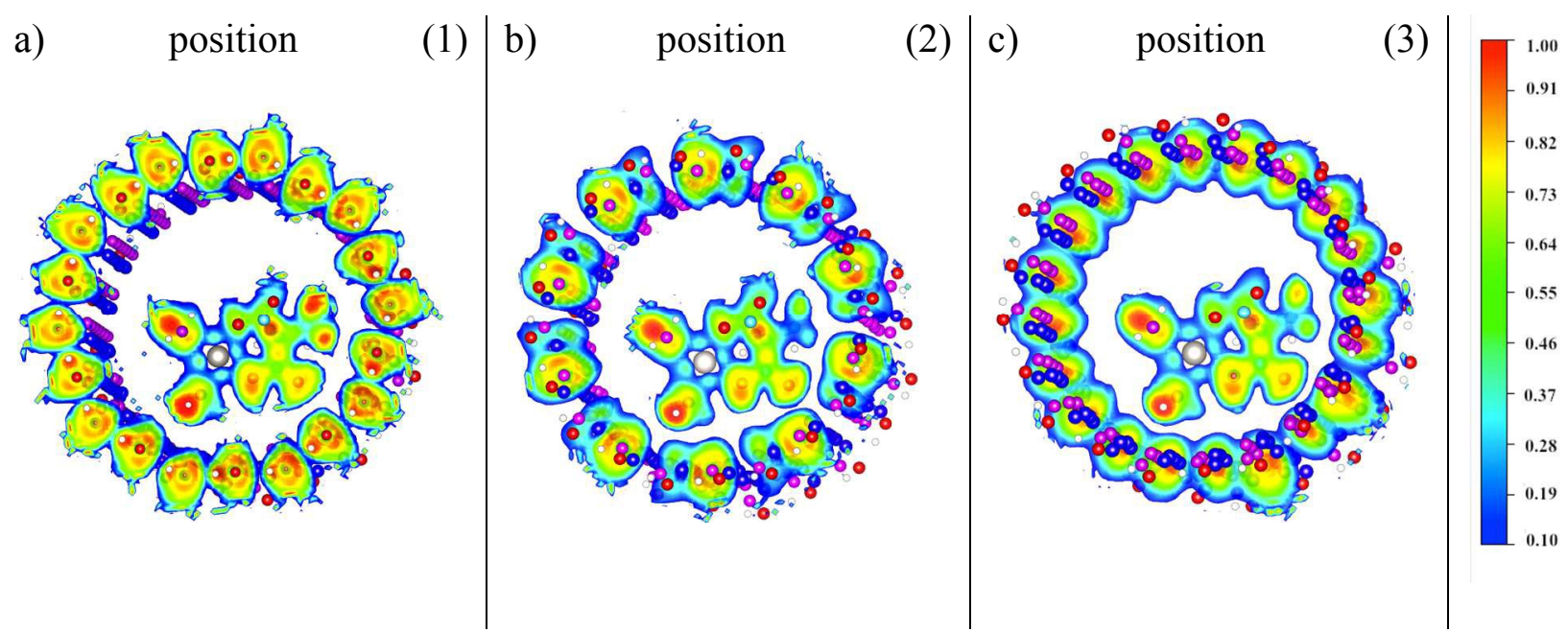

Figure 4: ELF representation in position (1), (2) and (3) of Figure 2. The values of each color are depicted in the diagram shown at right. The projection plane sketched are perpendicular to the $\mathrm{x}$ axis and centered onto the Pt atom. 


\subsection{Role of the solvent and of the dispersion repulsion contributions.}

To improve our studies we performed all-atom molecular dynamics simulation in water solvent using CHARMM36 parameter with Carboplatin/BNNT system, only. To do that, CPT was placed in front of the BNNT entrance (at about $6 \AA$ of the hydroxyl groups) and the total system was solvated in order to avoid periodic boundary interactions between each molecule. Then, the molecular dynamic simulations were launched without any constraint on the BNNT or the drug molecule. Figure 5a describes the course of the drug during the simulation, associated with its interaction terms with water and with the $\mathrm{BNNT}$. From $\mathrm{t}=0$ to $\mathrm{t}=2 \mathrm{~ns}$, we observe the thermalisation of the system. Then, the drug molecule is rapidly attracted by the nanopore (the beginning of the insertion (first green dashed line) is observed at $\mathrm{t}$ around $3.5 \mathrm{~ns}$ ) and stay inside (from $t=4.5 \mathrm{~ns}$, second green dashed line) during all the remaining time of the simulations (at least $10 \mathrm{~ns}$ ). This insertion is marked by a strong decrease of the interaction energy between the CPT and the BNNT nanopore (around $-1.8 \mathrm{eV}$, compared to $-1.15 \mathrm{eV}$ using DFT) while the contribution coming from the water molecules tends to be rather constant (about $-4.3 \mathrm{eV}$ ). Compared to the DFT results which did not take into account the dispersion repulsion terms and the presence of solvent, this result seems to be in quite good agreement with a stable encapsulation of the CPT. When the CPT is inside the BNNT, it diffuses on the inner surface without escaping until the end of the simulations. To better reproduce the curve showed in Figure 2, we plot in Figure $5 \mathrm{~b}$, the variation of the adsorption energy as a function of $\mathrm{d}(\mathrm{d}=0$ is for $\mathrm{Pt}$ atom located at the BNNT center of mass). This adsorption energy was calculated by subtracting to the total interaction energy those of the CPT alone in the water. Plotting the average of the sampling as a function of $\mathrm{d}$, we can distinguish the diffusion path of the CPT during its encapsulation. As for figure 2, the adsorption energy is characterized by an energy well $(\mathrm{E}=$ - 
$1.25 \mathrm{eV})$ far from the tube aperture $(\mathrm{d}=15 \AA)$. Then an energy bump appears when the molecule is close to the $-\mathrm{OH}$ group of the $\mathrm{BNNT}(\mathrm{E}=-0.45 \mathrm{eV} ; \mathrm{d}=\sim 10 \AA)$. It should be noted that this barrier is not retrieved in DFT and should be due to desolvation of the CPT to be encapsulated (see increase of the CPT-water energy contribution in Figure 5a since $\mathrm{t}=4 \mathrm{~ns}$ ). A profound energy well $(\mathrm{E}=-1.4 \mathrm{eV})$ is observed for $\mathrm{d}=9 \AA$ (as in Figure 2). Finally, the molecule should pass through a barrier $(\mathrm{E}=-1 \mathrm{eV})$ inside the BNNT $(\mathrm{d}=7.5 \AA)$ before being completely seized (d around $3 \AA$ ) with a stable energy $(\mathrm{E}=-1.35 \mathrm{eV})$. Note that the important sampling of the molecular dynamic simulations around $\mathrm{d}=3 \AA$ corroborates the stable encapsulation of the drug inside the BNNT.

a)

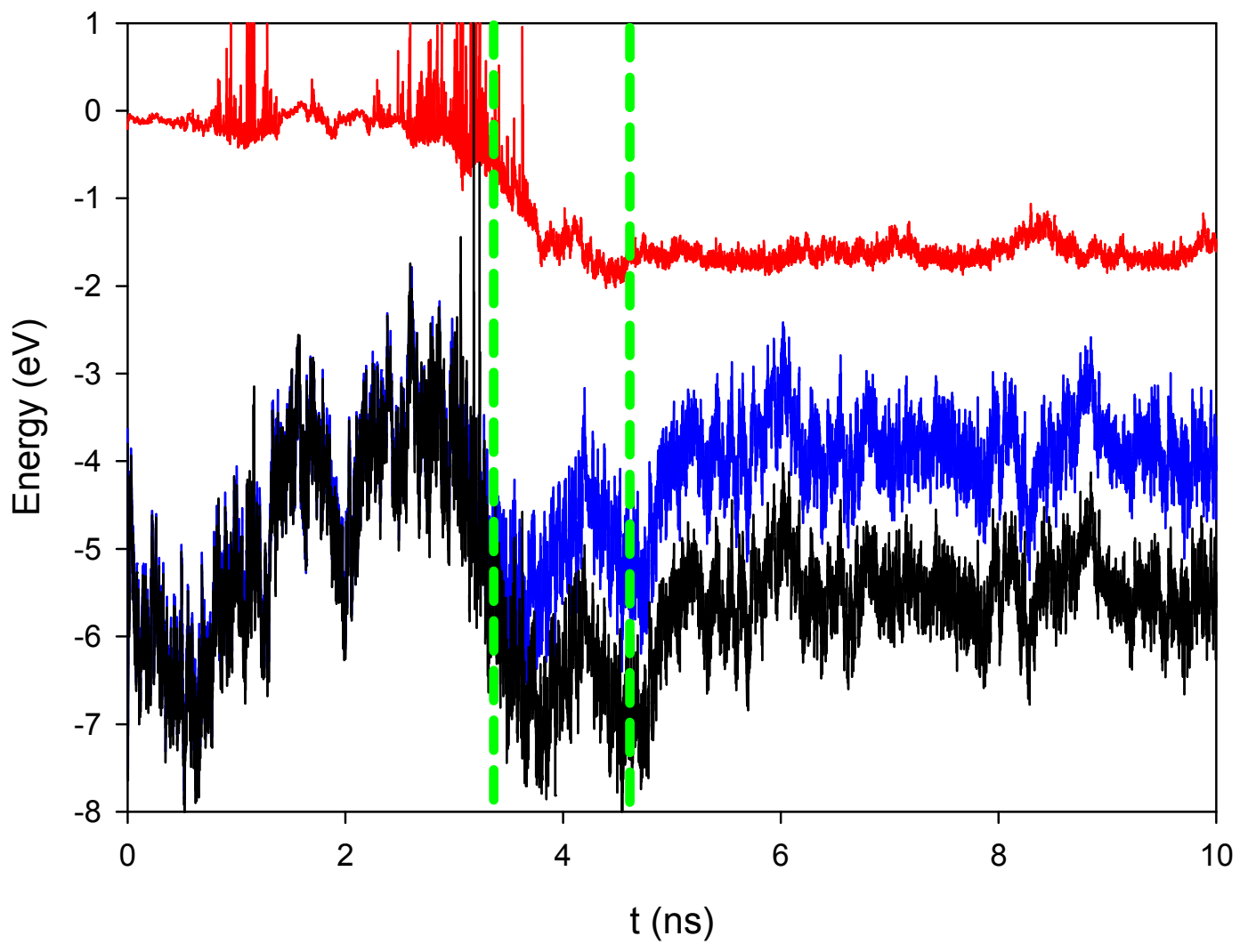


b)

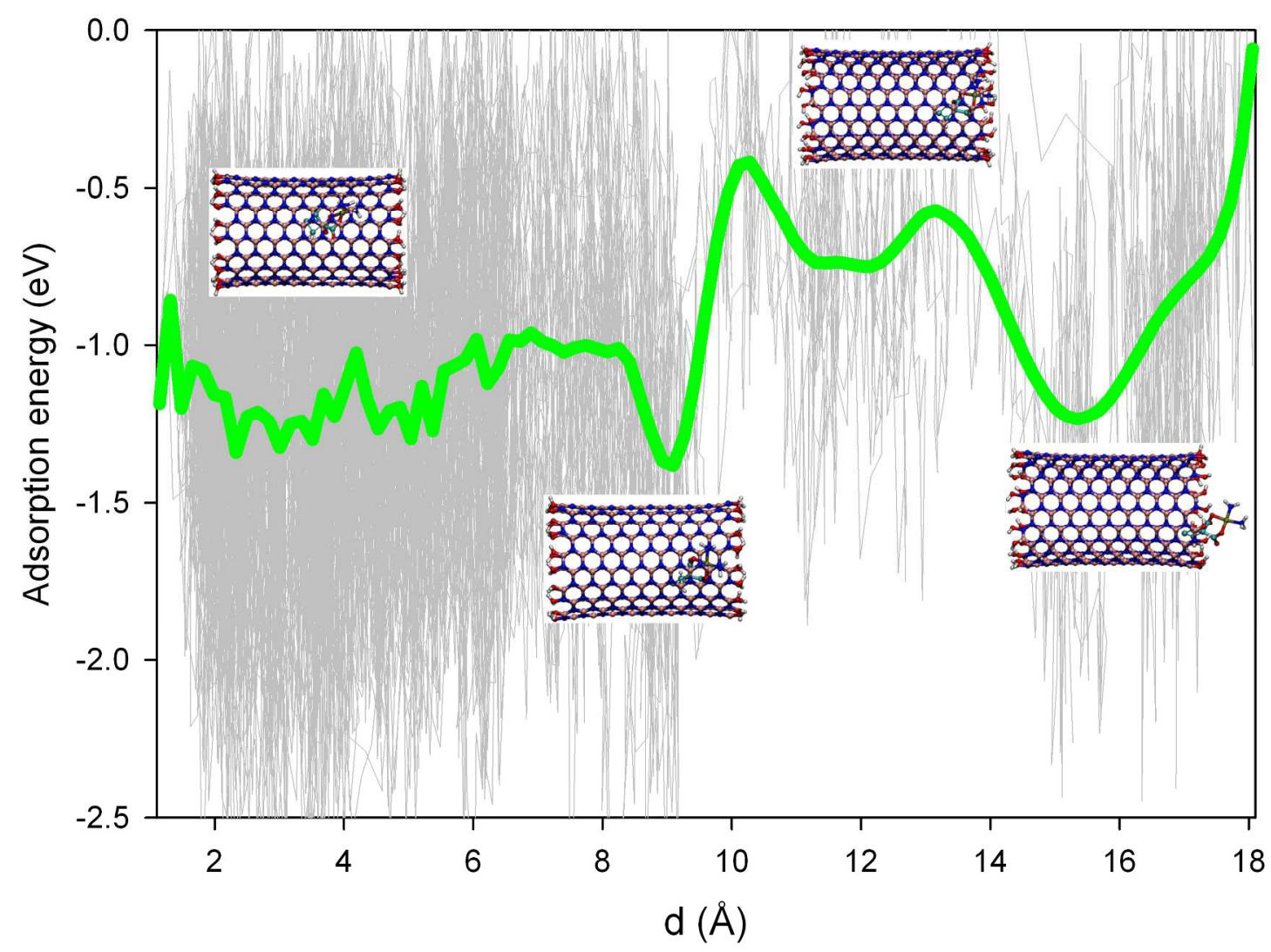

Figure 5: a) Interaction energy of CPT with BNNT (red curve) and with water (blue curve) during the whole molecular dynamic simulation in ns. Total energy is presented in black. The two vertical green dashed lines represent the beginning and the end of the molecule entrance. $b$ ) Total energy minored by the CPT interaction alone in water with distance $\mathrm{d}$.

\subsection{Discussion}

In this study, we show that BNNT could incorporate inside their internal cavity some molecular species. The very small variation of the electronic density of states and charges on the atom 
constituting the anticancer agent is due to the physical interaction between the molecule and the nanopore surface and could be explained by the low reactivity of the BNNT support. To compare, some calculations performed on carbon nanotubes showed that cycloaddition on the carbon surface could take place preferentially. In this specific case, the release of the therapeutic is difficult to achieve unless a specific external excitation arises (infra-red pulse for example). Concerning BNNT, we could expect that no supplementary electromagnetic field should be necessary to liberate the toxic molecules around the cancer cell. However, as shown in Figures 2 and $5 \mathrm{~b}$, the entrance of the BNNT is quite difficult to achieve thanks to a small barrier due to electrostatic interaction in DFT and desolvation when the molecule is placed in solvent (Figure 5b). However, the molecules tend to fall into a profound energy well (around $-1.15 \mathrm{eV}$ for CPT), which could be difficult to overcome in order to be delivered. These energy barriers fully dependent on the functionalized dangling bonds should lead to a thermal activation release of the anticancer drug molecule near the cell. This is confirmed by MD simulation in a solvent that did not show any release of the CPT once easily encapsulated. This corroborates the work performed by Panczyck et al. ${ }^{33}$ who found on a large SWCNT that the release of cisplatin was extremely fast (below few minutes) and necessitated only an energy barrier of $25 \mathrm{kcal} / \mathrm{mol}(1.08 \mathrm{eV})$. Other experiments developed by Li et al. ${ }^{34}$ on SWCNT (or Tripisciano et al. on MWCNT ${ }^{35}$ ) did not reach such velocity to release drug molecules. It needs around 6 hours to liberate at least $95 \%$ of the total encapsulated active molecules near the cancer cell. This is in fact a proof that the stability of the drug molecule inside the nanocarrier is very strong. Is should be remarked, nonetheless, that several defects could be at the origin of the large discrepancy observed between the theoretical and the experimental observations. However, our calculations estimate that the molecules should pass of an energy barrier of around $1 \mathrm{eV}$ to exit the BNNT nanotube, mainly 
due to the important dipole moment of the $\mathrm{CPT}^{33}$ Moreover, molecular dynamic simulations demonstrated that the mean energy barrier to kinetically exit from the BNNT is close to $0.8 \mathrm{eV}$ while the energies outside $(d=15 \AA)$ and inside $(d=9 \AA)$ the BNNT presented only a difference of $0.15 \mathrm{eV}$ (the most stable state being the encapsulated one). Increasing the energy barrier should increase the stability of the drug molecule inside the nanocarrier and such increase the release time of the drugs. Note that the residence time of the drug could be estimated depending on the energy barrier height. Assuming that the release is thermally active and following an Arrhenius law the CPT molecule could stay at least 2.7 hours in the BNNT. This should be read with care because the exponential law varies dramatically in the range of energy barrier calculated in our simulations. However, we never observed in the molecular dynamics simulations any desorption of the confined molecule. The diameter of the nanotube is also an important data that could lead to an important variation of the energy barrier and thus of the release time. Decreasing the tube diameter could better stabilize the molecule in the confined position, but would also increase the release time. The choice of the optimized diameter to obtain the best nanovector is still in course. Very recently, Muzy et al. performed release and treatment of cancer cell using confined cisplatin molecules inside carbon nanotubes. The role of the carbon nanotube diameter was studied in details and revealed that the larger diameter was more cytotoxic than the smaller one while the release time of cisplatin, increased in the high confinement state (smaller diameter) improved their anticancer efficacity. ${ }^{36}$

\section{Conclusion}

In this study, we have investigated interactions between boron nitride nanotube and carboplatin, combining full atoms DFT calculations and MD simulations. The outcomes were 
similar while the two theoretical methods were different. The platinum salt presented a strong oriented dipole moment that induced a very important dissymmetry in its energy path inside the BNNT. Moreover, we retrieved the same energy barrier near the entrance of the nanopore due to the functionalization of the BNNT dangling bonds with hydroxyl groups.

Incorporation of CPT molecules into BNNTs represents a good alternative for drug loading and hinders premature deactivation of CPT drugs before reaching the cancer cells. Moreover, encapsulating drugs within the interior cavity of BNNTs by means of physical interactions, liberates the external surface area of the nanovector for further chemical functionalization. Indeed, comparison of the density of states of the CPT molecule in the gas phase with the density of states of CPT incorporated inside BNNT shows slight discrepancies only.

The solvent effect and dispersion repulsion contributions taken into account using molecular dynamics simulations did not change our main results and confirmed that therapeutic agents were not affected by the surrounding water molecules when they were adsorbed inside BNNTs. All these results highlight a great potential in the exploitation of BNNTs in nanomedecine.

\section{Acknowledgments}

This present work was financially supported by University of Franche Comté in its "accueil de jeunes chercheurs en séjour de recherche post-doctorale" program.

Calculations were performed with the supercomputer regional facility Mesocenter of the University of Franche-Comté.

\section{References}


1. X. Yang, H. Hong, J. J. Grailer, I. J. Rowland, A. Javadi, S. A. Hurley, Y. Xiao, Y. Yang, Y. Zhang, R. J. Nickles, W. Cai, D. A. Steeber and S. Gong, Biomaterials, 2011, 32, 4151-4160.

2. C. Samori, H. Ali-Boucetta, R. Sainz, C. Guo, F. M. Toma, C. Fabbro, T. da Ros, M. Prato, K. Kostarelos and A. Bianco, Chem. Commun., 2010, 46, 1494-1496.

3. T. M. Allen and P. R. Cullis, Science, 2004, 303, 1818-1822.

4. M. Ferrari, Nat. Rev. Cancer, 2005, 5, 161-171.

5. K. K. Jain, Clin. Chem., 2007, 53, 2002-2009.

6. P. S. Kim, S. Djazayeri and R. Zeineldin, Gynecol. Oncol., 1016, 120, 393-403.

7. K. Kostarelos, A. Bianco and M. Prato, Nat. Nano., 2009, 4, 627-633.

8. J. Zhou and J. J. Rossi, Mol. Ther. Nucl. Acids, 2014, 3, e169.

9. R. Bawa, Nanomedicine, 2009, 5, 5-7.

10. Z. Liu, S. Tabakman, K. Welsher and H. Dai, Nano Res., 2009, 2, 85-120.

11. M. Prato, K. Kostarelos and A. Bianco, Acc Chem Res, 2008, 41, 60-68.

12. X. Blase, A. Rubio, S. G. Louie and M. L. Cohen, EPL (Europhysics Letters), 1994, 28, 335.

13. G. Ciofani, V. Raffa, A. Menciassi and P. Dario, J. Nanosci. Nanotech., 2008, 8, 6223-6231.

14. S. Y. Xie, W. Wang, K. A. Fernando, X. Wang, Y. Lin and Y. P. Sun, Chem Commun, 2005, 7, 36703672.

15. B. Akdim, S. N. Kim, R. R. Naik, B. Maruyama, M. J. Pender and R. Pachter, Nanotechnology, 2009, 20, 0957-4484.

16. E. Duverger, T. Gharbi, E. Delabrousse and F. Picaud, Phys. Chem. Chem. Phys., 2014, 16, 18425 18432.

17. G. Ciofani, S. Danti, G. G. Genchi, B. Mazzolai and V. Mattoli, Small, 2013, 9, 1672-1685.

18. G. Ciofani, S. Danti, S. Nitti, B. Mazzolai, V. Mattoli and M. Giorgi, Int. J. Pharm., 2013, 444, 85-88.

19. A. Celik-Aktas, J. M. Zuo, J. F. Stubbins, C. Tang and Y. Bando, Appl. Phys. Lett., 2005, 86, 133110(133111-133113).

20. Y. Gogotsi, Nanotubes and Nanofibers, Taylor \& Francis Group, 2006.

21. S. Kalay, Z. Yilmaz, O. Sen, M. Emanet, E. Kazanc and M. Culha, Beils. J. Nanotech., 2015, 6, 84102.

22. C. Y. Zhi, Y. Bando, T. Terao, C. C. Tang, H. Kuwahara and D. Golberg, Chemistry - An Asian Journal, 2009, 4, 1536-1540.

23. P. Hohenberg and W. Kohn, Phys. Rev., 1964, 136, B864-B871.

24. W. Kohn and L. J. Sham, Phys. Rev., 1965, 140, A1133-A1138.

25. J. P. Perdew, K. Burke and M. Ernzerhof, Phys. Rev. Lett., 1996, 77, 3865-3868.

26. P. Ordejon, E. Artacho and J. M. Soler, Phys. Rev. B, 1996, 53, R10441-R10444.

27. R. B. Best, X. Zhu, J. Shim, P. Lopes, J. Mittal, M. Feig and A. MacKerell, J. Chem. Th. Comput., 2012, 8, 3257-3273.

28. C. G. Mayne, J. Saam, K. Schulten, E. Tajkhorshid and J. C. Gumbart, J. Comput. Chem. , 2013, 34, 2757-2770.

29. N. J. Wheate, S. Walker, G. E. Craig and R. Oun, Dalton Trans., 2010, 39, 8113-8127.

30. L. Kelland, Nat. Rev. Cancer, 2007, 7, 573-584.

31. B. Silvi and A. Savin, Nature, 1994, 371, 683-689.

32. M. El Khalifi, E. Duverger, H. Boulahdour and F. Picaud, Anal. Methods, 2015, in press, DOI: 10.1039/C5AY00748H

33. T. Panczyk, A. Jagusiak, G. Pastorin, W. H. Ang and J. Narkiewicz-Michalek, J. Phys. Chem. C, 2013, 117, 17327-17336.

34. J. Li, S. Q. Yap, S. L. Yoong, T. R. Nayak, G. W. Chandra, W. H. Ang, T. Panczyk, S. Ramaprabhu, S. K. Vashist, F.-S. Sheu, A. Tan and G. Pastorin, Carbon, 2012, 50, 1625-1634. 
35. C. Tripisciano, K. Kraemer, A. Taylor and E. Borowiak-Palen, Chem. Phys. Lett., 2009, 478, 200205.

36. L. Muzi, C. Ménard-Moyon, J. Russier, J. Li, F. Chin, W. Ang, G. Pastorin, G. Risuleo and A. Bianco, Nanoscale, 2015, 7, 5383-5395. 


\section{The Table of Contents:}

The encapsulation of carboplatin anti-cancer drug is energetically favored inside small hydrated boron nitride nanotubes with a long release time.

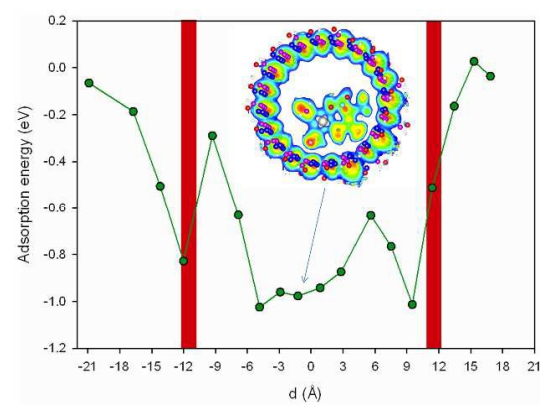

